\section{Response: Of Pigs and Primitive Notions}

\section{David Detmer \\ Purdue University/Calumet}

I agree with Gary Comstock's conclusion that the research program at Beltsville is not morally justified. Moreover, I have no substantial disagreement with the details of his clear, closely-reasoned argument.

However, it seems to me that two aspects of Comstock's argument call for further consideration and commentary, both because of their intrinsic philosophical interest and because they are the points in the argument most likely to be rejected by those predisposed to approve of the Beltsville project. I have in mind

1. Comstock's claim that "it is morally wrong, in the same way if not to the same degree, to deprive an animal of living conditions in which its basic biological needs can be met, as to deprive a human of living conditions in which its basic biological needs can be met," and

2. His appeal to the concept of a "primitive notion" as part of his defense of (1).

Let us begin with (1). I think nearly everyone would go along with Comstock to the point of agreeing that it would be wrong to prevent a pig from meeting its basic biological needs for no reason at all, or for some ignoble reason such as getting sadistic kicks from doing so, or for the purpose of achieving some fantastically inconsequential, though otherwise legitimate, human benefit. However, a very widespread opinion holds

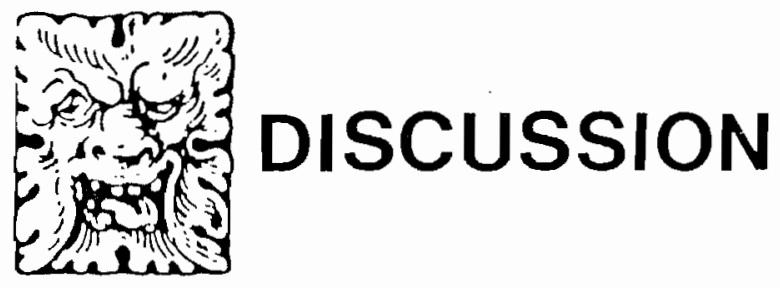

1. that pigs occupy a vastly lower moral status, and rightly command significantly less moral consideration and respect, than do human beings;

2. that nominally similar wrongs done to pigs and to humans are for that reason not at all morally comparable;

3. that it is therefore not morally wrong even in the same way, let alone to the same degree, to prevent a pig from meeting its basic biological needs as to visit nominally the same evil on a human being; and

4. that it is therefore perfectly acceptable to prevent a pig from meeting its basic biological needs in order to bring about a legitimate and not inconsequential benefit to human beings, even if this benefit is merely an economic one of the magnitude promised by the Beltsville program.

In short, according to this way of thinking, with its emphasis on the allegedly reduced moral status of pigs in comparison to that of humans, the Beltsville program is perfectly justified.

Comstock, of course, is aware that the issue of morally relevant differences between pigs and humans is crucial to his disagreement with his philosophical opponents. After all, immediately after arguing that no one would consider the economic benefits promised by the Beltsville program sufficient to render it morally permissible to treat humans the way pigs are treated in that program, he goes on to remark that "of course, the experimental animals are hogs, not humans." However, his worry in bringing this up is that "it is not apparent to everyone that hogs have what most children have, namely, the capacity to take an interest in their welfare." He seems to assume that if his opponents can be brought to see that pigs take the same sort of interest in having their own basic biological needs met as humans take in seeing their own biological needs met, these opponents will then have to acknowledge that the same sort of wrong is committed whenever a being which takes an interest in its welfare is prevented from meeting its basic biological needs, whether that being is a pig or a human. It is for this reason, apparently, that Comstock calls his claim that pigs do take an interest in their own welfare "the crux of my argument." 
My own sense, on the contrary, is that a good many people who quite readily concede that pigs have interests, and indeed who concede even that, at least when dealing with matters as elementary and uncomplicated as basic biological needs, pigs take the same kind of interest with respect to themselves as human beings do with respect to themselves, nonetheless feel that to thwart an animal's interest is not even close to being morally on a par with thwarting a human being's relatively similar interest. The reason is simply that animals, on the view I am now considering, occupy such a vastly reduced moral status in comparison to that of human beings that nominally similar wrongs done to animals and to humans should in fact be regarded as qualitatively different kinds of wrongs; indeed, if the "wrong" done to the animal is necessary to the furtherance of some legitimate human benefit, it should not, on this view, be considered a "wrong" at all.

One can distinguish two different strategies which have been used widely in attempts to justify the alleged radical difference between humans and other animals with regard to their respective levels of moral status. ${ }^{1}$ One strategy consists of attempting to isolate some feature or cluster of features held in common by all human beings, but not held by any nonhuman animals, and then claiming that any being who lacks this feature or cluster of features is, morally speaking, a secondclass citizen at best, or a moral nonentity at worst. Thus, various thinkers have appealed to such features as rationality, mastery of a language, possession of an immortal soul, the ability to reflect, and so on, either individually or collectively, as features a being must have in order to count substantially in moral deliberation and as features which, as it turns out, human beings, but no other animals, possess. ${ }^{2}$

In my judgment, this strategy cannot succeed. It faces at least two powerful and fundamental objections. The first of these has been much-discussed and is sometimes called "the problem of marginal humans." The problem is that the defender of this strategy faces a dilemma. Either the conditions to be met in order for a being to enjoy full moral status will be set high enough to exclude all nonhuman animals, in which case some severely mentally-enfeebled humans-those who are severely retarded or brain-damaged, for example-will fail to make the cut, or else the threshold will be set low enough to include all humans, in which case many animals will have to be included as well.
To be fair, I must acknowledge that several attempts to meet this objection have been put forward. For example, it has been argued that the features characteristically possessed by members of the group to which an individual belongs are of greater moral relevance than the features possessed by the individual in question or that we can supplement a high, individually-oriented, standard of inclusion into the realm of full moral status with an appeal to our natural sympathy for less fortunate members of our own species. ${ }^{3}$

I find these attempts miserably ad hoc and utterly implausible. To begin with the first attempt, surely it is not the case that features characteristically held within a group are of greater moral relevance than features actually held by the specific individuals in question. If tall people are typically better at basketball than short people, does that justify giving a basketball-playing job to a tall but inept basketball player over a short but highly talented one? If men are typically better than women at some task, does that justify hiring a specific man over a specific woman, when she, in this instance, can do the job more capably? And with regard to the other attempt to escape this difficulty, do we really want to let our natural sympathies play such an important role in our ethics? Here I think it is quite proper to make the much-criticized point that since we would never tolerate such a move with regard to justifying our differing treatment of individuals on the basis of their race or sex, we also should not do so in connection with the treatment of individuals on the basis of their species-membership. ${ }^{4}$

The other fundamental objection to the strategy of attempting to isolate some feature or set of features held in common by all humans but no nonhuman animals, so as to include the former and exclude the latter from the realm of full moral status, consists in questioning the moral relevance of the proposed feature or set of features. Consider, for example, the idea that what places human beings on a special moral plane is the fact that they, uniquely, can communicate in language. The obvious reply is that while this feature of human experience is morally relevant in some situations, it is obviously not in others. Thus, while the fact that a given pig can neither read nor write nor speak in a language provides an excellent reason to deny the pig admission to study in a university, it is far from clear how its linguistic capacities are relevant to the question of the moral legitimacy of causing the pig physical pain. Here, to cite once again Jeremy Bentham's famous line, "the 
question is not, Can they reason? nor, Can they talk? but, Can they suffer?"s

Some might conclude at this point that only a rather modest, animal-including criterion of inclusion into the realm of full moral status-for example, sentience, rather than linguistic competence or rationality-should be adopted, since it is of more obvious moral relevance to a greater number of situations than are its competitors. However, an alternative conclusion would be that there simply is no single feature, or single set of features, that is always exhaustively relevant in any situation to determining who should, and who should not, be given full moral consideration in that situation. The point is simply that the characteristics which are relevant to one situation are not always those which are relevant to another, so one cannot point to any single set of characteristics that some beings might share and declare them the unique and sufficient determiners of moral status. James Rachels explains this clearly:

A difference between individuals that justifies one sort of difference in treatment might be completely irrelevant to justifying another difference in treatment...[S]uppose the lawschool admissions committee accepts one applicant but rejects another. Asked to justify this, they explain that the first applicant had excellent college grades and test scores, while the second applicant had a iniserable record. Or suppose [a] doctor treats two patients differently: he gives one a shot of penicillin, and puts the other's arm in a plaster cast. Again; this can be justified by pointing to a relevant difference between them: the first patient had an infection while the second had a broken arm.

But now suppose we switch things around. Suppose the law school admissions committee is asked to justify admitting A while rejecting $B$, and replies that $A$ had an infection but $B$ had a broken arm. Or suppose the doctor is asked to justify giving A a shot of penicillin, while putting B'S arm in a cast, and replies that A had better college grades and test scores. Both replies are, of course, silly, for it is clear that what is relevant in the one context is irrelevant in the other. The obvious point is that, before we can determine whether a difference between individuals is relevant to justifying a difference in treatment, we must know what sort of treatment is at issue.... ${ }^{6}$

As a corollary to this conclusion, Rachels draws another: "namely, that there is no one big difference between individuals that is relevant to justifying all differences in treatment."7 It seems to me that this provides just what we need to show that Comstock is right to conclude that the wrong done to pigs when they are prevented from having their basic biological needs met is similar, if not identical, to the wrong done to human beings when they are made to suffer that fate. Comstock's opponents will want to argue that the difference in moral status between pigs and human beings is crucial here and, at least according to the strategy which I am now considering, that this crucial difference in moral status stems from differences in rationality, or linguistic competence, or the like, between the two species. However, as the argument borrowed from Rachels clearly shows, no such move can possibly succeed unless these admitted differences between pigs and humans can be shown to be relevant to the issue of the thwarting of basic biological needs. But the very fact that these needs are basic_-not advanced and thus requiring advanced capacities - and biological - rather than cultural or intellectual-indicates that the advanced capacities which distinguish most humans from pigs are of minimal relevance to the moral evaluation of the treatment handed out in the Beltsville program. The harm done to the Beltsville pigs hurts them in a way that is quite comparable to the way in which such treatment would hurt humans, were they subjected to it. And this treatment violates the interests of the Beltsville pigs much in the same way that it would violate the interests of comparably-treated humans. Thus, since there is probably a consensus that such treatment would be indefensible if directed at humans, how can it be defended in connection with pigs?

This brings me to the second of the two major strategies which have been widely used in attempts to defend the idea that nonhuman animals should be regarded as occupying only a radically reduced level of moral status in comparison to that enjoyed by humans. This strategy consists of the simple declaration that the rightness of assigning a privileged moral status to human beings is intuitively evident - that it possesses a kind of deep obviousness which renders argument unnecessary and that it certainly possesses a greater 
degree of obviousness than does any of the proposed arguments attempling to overturn it. To be sure, one might ask for supporting arguments anyway, in spite of the alleged obviousness of the claim in question. But if no such arguments can be found which can stand up to scrutiny, this need not inply that we should abandon the claim, and this is so because of the very logic of justification. So what is to stop us from claiming that the moral rightness of giving radical priority to human interests over those of other animals whenever they conflict is simply intuitively evident, a "primitive notion," to use Comstock's phrase? Notice that if we can legitimately make this move, we presumably have all we need in order to defend the Beltsville program. But can we legitimately make this move?

It is important to note that Comstock is not in a position to make a quick, principled rejection of this move, since he makes a similar one on behalf of his claim that "it is wrong to deprive a being that can take an interest in having its basic biological needs met of the things it needs to have its basic biological needs met." Comstock asserts that this latter claim is a "primitive notion," by which he apparently means a notion that is so basic and fundamental that it cannot be grounded or justified by appealing to anything else even more basic or fundamental, but which is at the same time so obvious that nothing beyond mere clarification and vivification is needed to render the notion acceptable to all minimally rational and welladjusted persons.

My own argument in support of Comstock's use of the concept of "primitive notions," and one which I think is consistent with the spirit of his own defense of it, runs as follows. If I tell you that I believe A, you might justly ask me why, in which case I would invoke B in support of A. But suppose that you do not readily accept $B$. Then I could cite $C$, and argue that it implies $B$. Should you then prove unwilling to accept $C$, I might appeal to D, and so on, but clearly this must come to a stop somewhere. It would seem, then, that for any argument to get off the ground it must be possible either to assume or eventually to find starting points which all parties to the discussion can accept. I cannot see any way around this analysis. Indeed, it seems to me that it holds for all arguments whatever, on all subjects. The only reason we are not more aware of this, I suggest, is that a practical matter we all do indeed share a large number of "obvious" assumptions in common and that these rarely emerge as objects of explicit attention in our discussions and arguments with one another. We do not notice these assumptions because we are rarely motivated to question them or refer to them, their obviousness being such that neither we nor our interlocutors are often called on to question them, and their basicality being such that they are remote from the higher-level, more complex disputes which usually engage our attention.

But in the context of the present debate, this appeal to intuition, or to "primitive notions," has to be addressed since the appeal is being made on behalf of opposite conclusions. Recall that, for Comstock, it is a primitive notion that "it is wrong to deprive a being that can take an interest in having its basic biological needs met of the things it needs to have its basic biological needs met." Since pigs can take an interest in having their basic biological needs met, it follows that the Beltsville program is wrong. But other thinkers invoke intuition in order to argue that human interests are what really count from a moral standpoint, even if we cannot give any cogent argument on behalf of denying similar consideration to animals' interests. For example, consider the words of C. A. J. Coady in his essay, "Defending Human Chauvinism":

It will be said that the idea that there is something specially morally important about human beings needs justification... but I'm not sure that it has to be [justified]. There are various ground floor considerations in ethics as in any other enterprise-for an animal liberationist such things as the "intrinsic good" of pleasure and the "intrinsic evil" of pain are usually ground floor. No further justification is given for them, or needed. It is not clear to me that membership in the human species does not function in a similarly fundamental way in ethics so that there is as much absurdity, if not more, in asking "Why does it matter morally that she is human?" as in asking "Why does it matter morally that she is in pain?"8

How, then, do we choose between Comstock's primitive notion and Coady's ground floor consideration? Are the two on equal footing? Must our choice between them be made simply arbitrarily?

I think not. I come down firmly on Comstock's side, and I do not consider my choice the slightest bit arbitrary. By way of explanation, I offer three points. 
First, it does indeed seem to me powerfully evident, from my own experience and from reflection on that experience, that, for example, happiness is intrinsically good and misery is intrinsically bad, or at least that happiness is intrinsically better than misery. Can I give an argument in support of this valuation, deducing it from more fundamental principles or theories? No, it seems to me that my valuation is intuitive; or, if you prefer, it is a primitive notion or a ground floor consideration. Do I have a comparably strong intuition supporting the idea that human interests should be granted a privileged moral status in comparison to animals' interests? I do not. For one thing, when I reflect on my experientially-grounded sense of the badness of my misery, it appears to me quite clearly that the badness flows from the nature of misery, rather than from the fact that I am the one experiencing the misery. To be sure, the fact that I am the one experiencing the misery explains why I am the one who is intimately aware of this particular case of badness. But the misery appears to me to be bad as such-something which would be bad for whoever experienced it, including, for example, a pig. Of course, one could maintain that a pig in fact cannot experience such inisery, but that is another issue, and one which, in my judgment, Comstock answers admirably. Alternatively, one might present some argument for the conclusion that, while pigs do indeed experience the same sort of misery that I experience, it is not nearly so bad a thing for the pig to experience it as it is for me to do so. But this would require argument. Since what is clearly given in my experience of misery is that the experience of misery as such is seriously bad, the burden of proof is on those who would claim that sometimes it is not. Given this burden of proof, intuition, in the absence of argument, will not be sufficient.

My second point in support of Comstock's primitive notion over Coady's ground floor consideration stems from the observation that one of the chief practical dangers attaching to any appeal to intuition concerns the ever-present possibility that one is confusing the intuitively evident with the merely fainiliar or the culturally given. And yet, surely my experientiallygrounded conclusion that happiness is intrinsically better than misery flourishes independently of cultural support and, indeed, would flourish in the face of cultural opposition. For happiness and misery do not present themselves with the kind of plasticity or flexibility necessary for their evaluation to be strongly susceptible to cultural manipulation. This does not seem to me to be the case with regard to the issue of the degree of importance to which considerations of animals" interests should be given in our moral deliberations. Consider that, while it would be quite shocking to learn of a culture in which misery is greatly prized for its own sake, it does not even surprise us to read of cultures which assign to animals a radically different moral status, whether higher or lower, than that which we assign to them.

Finally, I would point out that while our opposed fundamental judgments - that misery as such is bad and that it is seriously bad only when suffered by humansare both widely held, it is possible in the case of the latter, but not the former, to explain the popularity of the judgment by appealing to something other than its evidentness. I refer to the simple point-another to which intuitionists would be well-advised to pay persistent attention - that the latter judgment, unlike the former, is self-serving, benefitting the relatively powerful humans who make the judgment, at the expense of the relatively powerless animals, who have no say in the matter. ${ }^{9}$

In conclusion, I reaffirm my acceptance of Comstock's conclusion and of the major details of his argument. I have here attempted only to strengthen that argument by defending two of its most controversial moves against the objections they are likely to generate in the minds of those who would defend the Beltsville research program.

\section{Notes}

${ }^{1}$ I add in passing that many people, perhaps most, simply accept and apply in their lives this extreme double standard; without ever undertaking to justify it, even to themselves. It is simply a part of the inherited moral code in many cultures, and a great number of people evidently never find a motive to question it.

${ }^{2} \mathrm{Cf}$. the selections by Aquinas, Descartes, and Kant in Tom Regan and Peter Singer, eds., Animal Rights and Human Obligations, 2nd ed. (Englewood Cliffs, NJ: Prentice Hall, 1989).

${ }^{3}$ For an example of the first attempt, see Carl Cohen, "The Case for the Use of Animals in Biomedical Research,". in Robert M. Baird and Stuart E. Rosenbaum, eds., Animal Experimentation: The Moral Issues (Buffalo, NY: Prometheus, 1991), pp. 103-114. For examples of the second attempt see Mary Anne Warren, "Difficulties with the Strong Animal Rights Position," in Baird and Rosenbaum, op. cit., 
pp. 89-99; and Bonnie Steinbock, "Speciesism and the Idea of Equality," in Jeffrey Olen and Vincent Barry, eds., Applying Ethics, 3rd ed. (Belmont, CA: Wadsworth, 1989), pp. 412-418.

${ }^{4}$ Cf. Peter Singer, "All Animals Are Equal," in Regan and Singer, op. cit., pp. 73-86; and James Rachels, Created From Animals (New York: Oxford University Press, 1990), pp. 175-197.

5 Jeremy Bentham, from The Principles of Morals and Legislation, Chapter XVII, Section 1, in Regan and Singer, op. cit., p. 26.
${ }^{6}$ Rachels, op. cit., pp. 177-178.

${ }^{7}$ Ibid., p. 178.

${ }^{8}$ C. A. J. Coady, "Defending Human Chauvinism," in Sylvan Barnet and Hugo Bedau, eds., Current Issues and Enduring Questions, 2nd ed. (Boston: Bedford Books, 1990), p. 265.

${ }^{9} \mathrm{Cf}$. Joel Feinberg, "Human Duties and Anima1 Rights," in his Rights, Justice, and the Bounds of Liberty (Princeton, NJ: Princeton University Press, 1980), pp. 185-206.

\section{Call for Papers: Eastern, Pacific, Central}

The Society for the Study of Ethics \& Animals meets in conjunction with the Division meetings of the American Philosophical Association. Papers are hereby invited for all 1994 meetings: Pacific in Late March, Central in late April, and Eastern in late December.

Papers are welcome on any topic connected with ethical issues affecting nonhuman animals. Possible topics include:

- The moral (in)significance of being natural (rather than domesticated or genetically engineered).

- The consonance (or lack thereof) of Animal Liberation Front actions with an animal rights ethic.

- Historical studies of conceptions of the moral standing of animals.

- Animals and the action ethics/virtue ethics distinction.

- The importance of animal issues to philosophy and philosophers.

- Companion animals and paternalism.

- The ethics and epistemology of animal research.

- and so on.
A system of blind refereeing is used. Papers must be ten to fifteen pages long, double spaced. Three copies are required, with the author's name and any other identifying information on a separate title page.

\section{Send papers to:}

\section{Harlan B. Miller, Executive Secretary}

Society for the Study of Ethics \& Animals c/o Department of Philosophy

Virginia Polytechnic Institute and State University Blacksburg, VA 24061-0126

Be sure to indicate which meeting is desired. Volunteers to comment on papers should submit their names to the same address. Deadlines (postmark) are as follows:

1994 Pacific meeting

1994 Central meeting

September 1, 1993

1994 Eastern meeting

September 1, 1993

March 15, 1994

Papers accepted for meetings will automatically be considered for publication in Between the Species. 